\title{
Reaction Rate Theory: Summarising Remarks
}

\author{
David Chandler \\ Department of Chemistry, University of California, Berkeley, California 94720, USA \\ David E. Manolopoulos \\ Department of Chemistry, University of Oxford, \\ Physical and Theoretical Chemistry Laboratory, \\ South Parks Road, Oxford OX1 3QZ, UK
}

\begin{abstract}
This paper summarises the contributions to the Faraday Discussion on reaction rate theory. The topics range from contemporary usage of transition state theory, including rare event sampling, to instantons and non-adiabatic dynamics.
\end{abstract}




\section{INTRODUCTION}

We would like to begin by thanking Professor Althorpe and his co-organisers for bringing together such a wide-ranging Discussion about various aspects of modern reaction rate theory. Since the discussion has been so wide ranging, we shall not attempt to summarise it all, but rather highlight some common themes that have emerged from it and give our perspective on them.

\section{TRANSITION STATE THEORY}

Chemical reaction rate theory began with the development of transition state theory (TST) by H. Eyring, E. Wigner, M. Polanyi and others in the 1930s, and there is still no better introduction to the subject than their combined contributions to the 67th General Discussion of the Faraday Society ${ }^{1}$ that was held in Manchester in 1937.

Of these contributions, Eyring's second paper on "the theory of absolute reaction rates" would probably be the most familiar to modern chemists. ${ }^{2}$ This paper describes both the statistical mechanical version of (separable) TST with harmonic oscillator vibrational and rigid body rotational partition functions for bimolecular chemical reactions in the gas phase, and the thermodynamic formulation of TST for activated chemical reactions in solution. Eyring did not have access to a computer, but he was nevertheless able to do some impressive calculations with these simple theories, which are still taught to undergraduate chemists for this reason to this day $\underline{3}$

In the modern computer age, Eyring's version of TST for gas phase bimolecular reactions has evolved into more sophisticated methods, such as the "canonical variational transition state" (CVT) theory ${ }^{4}$ Here the variational aspect consists of optimising the transition state dividing surface so as to minimise the TST rate, an idea that was first suggested in the classical context by Wigner ${ }^{\sqrt[5]{5}}$ and was highlighted by Keck in the $1960 \mathrm{~s}{ }^{[6}$ The CVT method is typically implemented with various semiclassical tunnelling corrections, which again have their origin in the work of Wigner (see below). These are described in the present contribution by Kästner and co-workers, ${ }^{7}$ who use a small curvature tunnelling correction to explain the experimentally observed increase in the rate of the astrophysically important $\mathrm{NH}_{3}^{+}+$ $\mathrm{H}_{2} \rightarrow \mathrm{NH}_{4}^{+}+\mathrm{H}$ reaction at low temperatures. 
Other contributions to the present Discussion that build directly on Eyring's work include the study of Jónsson and co-workers, ${ }^{8}$ who combine a geodesic nudged elastic band minimum energy path with harmonic TST to study the magnetisation transition of an iron island on a tungsten surface, and the study of Leliévre and co-workers, $\frac{9}{9}$ who use the concept of a quasi-stationary distribution within a metastable well to analyse the validity of mapping the Langevin dynamics of a problem with many metastable minima onto a Markov state model in which the jump rates between neighbouring minima are given by harmonic TST (here called the "Eyring-Kramers" formula because of the Langevin aspect - the paper by Ianconescu and Pollak on the Kramers turnover theory of surface diffusion also focusses on Langevin dynamics $\frac{10}{3}$.

In his Spiers Memorial Lecture at the 110th Faraday Discussion in 1998, Miller presented an alternative account of the 1937 Discussion, 11 in which he highlighted Wigner's contribution on "the transition state method" 12 This contribution began with a perceptive categorisation of reaction rate theory into what Miller describes as "three threes" 11 The first of these was that there are three steps in the theory of kinetics: finding potential energy surfaces, calculating elementary reaction rates, and solving a system of rate equations for the overall reaction. This is just as true today as it was 80 years ago, and it is nicely illustrated by the paper on gas phase polycyclic aromatic hydrocarbon (PAH) formation in the present Discussion by Mebel and co-workers. $\frac{13}{13}$ The second of Wigner's threes was that elementary collisions could be classified as ro-vibrationally inelastic, reactive, or electronically non-adiabatic, and the third was the three assumptions of (classical) TST: electronic adiabaticity, the validity of classical mechanics, and the existence of a dividing surface in configuration space that trajectories do not re-cross.11

Wigner then proceeded to discuss classical TST in more detail, and to argue that there were two fundamental obstacles to constructing a quantum mechanical version of the theory (QTST): the lack of a quantum mechanical analogue of the classical configurational Boltzmann factor, and the fact that the uncertainty principle precludes specifying the velocity along the reaction coordinate when the system is confined to the transition state dividing surface. He ended by pointing out that it was nevertheless possible to dress up classical TST with quantum corrections, and presented a semiclassical tunneling correction in his paper as an example. 12

In retrospect, Wigner's dynamical perspective has proved to be more fruitful than 
Eyring's statistical perspective in finding ways to go beyond TST. We now know, for example, that the classical TST rate is just the zero-time limit of an expression involving a classical reactive flux (i.e., flux-side) correlation function, the transient- or plateau ${ }^{14}$ time value of which gives the exact classical reaction rate coefficient. We also know from linear

response theory ${ }^{15}$ and subsequent analysis, $\frac{16}{16}$ that this classical flux-side correlation has a natural quantum mechanical generalisation that gives the exact quantum mechanical rate coefficient after a transient time. In bimolecular problems, the transient-time value can be taken as a long-time limit. But in reality the transient time is relatively short. It is the time for a system residing in the vicinity of the dynamical bottleneck to commit to one or the other stable basin. This feature has a direct bearing on the feasibility of rare-event sampling in complex systems because it reduces treatments of long-time dynamics to averages over short-time trajectories, about which we have more to say later.

Considerable progress has also been made with developing improved tunnelling corrections, the semiclassical "instanton" approximation ${ }^{17}$ being the definitive example (see below). And in spite of Wigner's objections, useful progress has in fact been made in constructing a QTST. There have been many attempts to do this, based on semiclassical arguments which Wigner was well aware of, $\frac{18 \mid 19}{19}$ and arguments involving imaginary time path integrals which he was not. 20121 These provide a natural quantum mechanical analogue of the classical configurational Boltzmann factor, and they also automatically include thermal uncertainty broadening. ${ }^{22}$ The most recent attempt to derive it using a combination of semiclassical and imaginary time path integral arguments has come to the conclusion that QTST is simply the short-time limit ${ }^{23} \sqrt[26]{26}$ of ring polymer molecular dynamics (RPMD) reaction rate theory $\sqrt{27+30}$ (just as classical TST is the short-time limit of classical reaction rate theory).

\section{INSTANTONS}

An instanton is a periodic orbit in imaginary time, or equivalently a periodic orbit in real time on the "upside down" potential! 17 The classical action around this periodic orbit is proportional to the WKB tunnelling integral at the energy $E$ of the orbit, and can therefore be used to calculate the microcanonical reaction probability $P(E)$ at any energy in the tunnelling regime (below the top of the reaction barrier). For a multidimensional reaction, there is no need to specify a tunnelling path, as this is provided automatically by the 
instanton. The reaction probability at any energy above the top of the reaction barrier can be calculated separately using the parabolic barrier approximation (which includes the effect of quantum reflection).

The thermal rate coefficient is proportional to a Boltzmann average of the microcanonical reaction probability. When the integral over $E$ in the Boltzmann average is evaluated using the steepest descent approximation, one finds that the rate coefficient at temperatures below $T_{c}=\hbar \omega_{b} / 2 \pi k_{\mathrm{B}}$ (where $i \omega_{b}$ is the imaginary frequency along the reaction coordinate at the barrier maximum) is given in terms of the action around an instanton orbit with period $\beta \hbar$ (where $\left.\beta=1 / k_{\mathrm{B}} T\right) ! 17 \mathrm{~A}$ discretised version of this thermal instanton orbit is a saddle point on the ring polymer potential energy surface, and this fact provides a direct connection between instanton theory and RPMD reaction rate theory 31

The instanton approximation to the thermal rate coefficient is very accurate at temperatures well below $T_{c}$, but it diverges as $T_{c}$ is approached from below. The parabolic barrier approximation to the thermal rate coefficient is accurate at temperatures well above $T_{c}$, but it diverges as $T_{c}$ is approached from above. The combination of the thermal instanton rate below $T_{c}$ and the thermal parabolic barrier rate above $T_{c}$ therefore breaks down in both directions in the vicinity of the crossover temperature.

Richardson's contribution to the present Discussion suggests a fix to this problem. 32 Rather than using the thermal instanton and parabolic barrier expressions, he recommends combining the instanton approximation to the reaction probability $P(E)$ at energies below the barrier maximum with the parabolic barrier approximation to $P(E)$ at energies above the barrier maximum, and doing the integral over $E$ in the Boltzmann average numerically rather than via the steepest descent approximation. This procedure is shown to work very well for the simple model problems that Richardson considers (including an Eckart barrier in one dimension and an uncoupled harmonic oscillator in another), $\frac{32}{2}$ but it remains to be seen how well it will work for more complicated reactions.

An example of a more complicated problem that challenges the instanton approximation is the $\mathrm{NH}_{3}^{+}+\mathrm{H}_{2} \rightarrow \mathrm{NH}_{4}^{+}+\mathrm{H}$ reaction considered by Kästner and co-workers. ${ }^{7}$ The instantons in this reaction undergo a symmetry breaking transition as the temperature is lowered, and they become unstable in the vicinity of this transition. Moreover the reaction has a "submerged barrier", as a result of which the thermal instanton orbit with period $\beta \hbar$ lies below the reactant asymptote at $E=0$ at all temperatures in the tunnelling regime 
$\left(T<T_{c}\right)$. This clearly invalidates the steepest descent approximation to the Boltzmann integral upon which the thermal instanton approximation is based, and it precludes the use of this approximation to calculate the bimolecular reaction rate. ${ }^{7}$

A situation in which instanton theory works much better is presented in the contribution of Jónsson and co-workers. ${ }^{8}$ In the second part of their paper, these authors derive an expression for the instanton crossover temperature $T_{c}$ of a spin with a Landau-Lifshitz action in an angular potential $U(\theta, \phi)$, finding that it has the same form $T_{c}=\hbar \omega_{b} / 2 \pi k_{\mathrm{B}}$ as in the standard (Cartesian) barrier crossing case but with $\omega_{b}$ now being a function of all three second derivatives of $U(\theta, \phi)$ at the barrier maximum. The resulting formula is used to calculate the crossover temperatures of various molecular magnets with both two- and fourfold anisotropy. Particularly impressive agreement is obtained with experimental results for the rate of the magnetisation transition in a $\mathrm{Mn}_{4}$ magnet, both in terms of the harmonic TST description of the Arrhenius behaviour above $T_{c}$ and in the location of $T_{c}$ itself (below which the experimental transition rate simply plateaus). The simple combination of harmonic TST and instanton theory captures all that matters in this system.

\section{NON-ADIABATIC DYNAMICS}

Many of the contributions to the present Discussion are concerned with electronically non-adiabatic dynamics. The most striking thing about them is that they present almost as many different approaches to the problem as there are contributions! This clearly reflects the fact that the problem is hard, and that there is as yet no completely general and reliable simulation tool for non-adiabatic dynamics in complex systems.

The simplest and most popular approach to non-adiabatic dynamics is surface hopping,

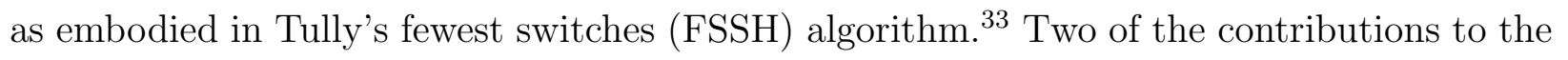
present Discussion use this algorithm, in both cases in conjunction with "on the fly" ab initio calculations of the relevant potential energy surfaces and non-adiabatic couplings. Szabla and co-workers use FSSH to study the photorelaxation of imidazole and adenine in water clusters, $\frac{34}{34}$ and Blumberger and co-workers apply it to a model molecular electron transfer (ET) reaction. .35

The latter of these two papers shows that the algorithm is capable of qualitatively capturing the correct non-adiabatic to adiabatic transition in the ET rate as the ratio $H_{\mathrm{ab}} / \lambda$ 
increases (where $H_{\mathrm{ab}}$ is the average diabatic electronic coupling strength and $\lambda$ is the reorganisation free energy), and also the transition to thermally damped Rabi oscillations at larger $H_{\mathrm{ab}} / \lambda$. Even the simulated transition to the Marcus inverted regime is plausible, although in this case there is a lot of noise in the FSSH results. $\stackrel{\sqrt[35]{5}}{{ }^{2}}$ While these results are encouraging, one should bear in mind that surface hopping is restricted to the regime in which the nuclear motion can be treated classically, $\underset{36}{6}$ there are issues with the treatment of energetically forbidden electronic hops, and the method suffers from a well-known over-coherence problem $^{37}$ to which there is no unique solution. $38+40$

The symmetrical quasi-classical (SQC) method discussed by Miller in his Introductory Lecture to this Discussion provides an interesting alternative to FSSH that is free from the over-coherence problem. $\stackrel{41}{ }$ This method has now been applied to a wide variety of model systems with impressive results, especially in its latest incarnation with triangular windowing functions. ${ }^{42}$ However, it has yet to be applied to a realistic molecular system with ab initio electronic potential energy surfaces, which might prove more difficult to deal with than those encountered in model systems. Strongly repulsive electronic states in particular can lead to unphysical forces on the nuclei when they have negative actions (populations)! 43 This problem will need to be fixed before the method can be used as a general simulation tool. And like FSSH, the present formulation of the SQC method neglects quantum mechanical effects in the nuclear motion. As the general SQC approach looks promising, it would certainly be worth trying to address these two issues.

Two methods that do include nuclear quantum effects are the non-adiabatic generalisations of RPMD discussed by Kretchmer and Miller $\stackrel{44}{4}$ and by Duke and Ananth. ${ }^{45}$ The first of

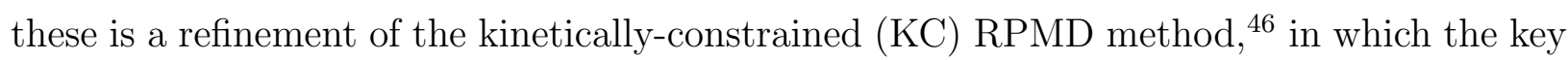
quantum mechanical interference effect in ET reactions (the requirement for the diabatic electronic states to be degenerate when the electron hops) is built into a two electronic state generalisation of RPMD as a constraint. The second is an alternative method for calculating ET rates that combines a particular form of non-adiabatic path integral TST with a "mean field" RPMD transmission coefficient. The results of both methods look encouraging. For example, they are both shown to capture the transition to the Marcus inverted regime with increasing thermodynamic driving force. ${ }^{44 \mid 45}$ However, it seems unlikely that either will capture the thermally damped Rabi oscillations in the electronic state populations that arise when the electronic coupling strength exceeds half the reorganisation energy ${ }^{35}$ And while 
KC-RPMD appears to provide quite a general simulation method for two electronic state problems away from this regime,,$\sqrt{44}$ this is unlikely to be the case for a method that uses different path integral formulations for the TST rate and the transmission coefficient .45

There are three further papers on non-adiabatic dynamics in the present Discussion, each of which presents yet another approach to the problem. The paper by Makri莐 explores a blip-summed method for evaluating a quantum-classical path integral (QCPI) in which the "system" path is treated quantum mechanically while the corresponding "bath" path follows a classical trajectory. The paper argues that significant computational savings over the standard QCPI approach can be achieved when the bath is composed of harmonic oscillators, and goes on to demonstrate the fact for a spin-boson problem with Ohmic spectral density. However, the standard QCPI approach has already been applied to systems with anharmonic baths, such as the ferrocene-ferrocenium charge transfer reaction in liquid hexane, $\frac{48}{4}$ and it remains to be seen whether the new blip-summed method can be adapted to study such systems.

The paper by Hele and Ananth ${ }^{49}$ derives the exact non-adiabatic quantum propagator (Liouvillian) in the Meyer-Miller ${ }^{[50151}$ Stock-Thos\$ ${ }^{52153}$ mapping variable representation, and shows how various well-known approximations to non-adiabatic dynamics such as the linearised semiclassical initial value approximation (LSC-IVR) or "classical Wigner model" can be obtained from it by dropping various terms. Unfortunately, these approximations lead to non-stationary processes: their dynamics does not conserve the quantum mechanical equilibrium distribution. This is a serious drawback for all but short time scale simulations of complex systems, for a variety of reasons including the zero point energy leakage problem. 54 And while it would be good if it could be done, it is not obvious how to obtain an approximation without this drawback by dropping terms from the exact Liouvillian.

Finally, the paper by Soudackov and Hammes-Schiffe ${ }^{[55}$ describes an approach to protoncoupled electron transfer (PCET) reactions that begins by using appropriate criteria to determine whether the reaction is sequential or concerted, and in the latter case whether it is in the adiabatic ( $\mathrm{H}$ atom transfer) or the non-adiabatic (electron-proton transfer) regime. An appropriate specialised theory is then used to study the situation at hand. This approach is applied to what is deemed to be a concerted, non-adiabatic, electron-proton transfer reaction in the enzyme soybean lipoxygenase (SLO). Here the appropriate theory is a Golden Rule expression for the PCET rate, which involves the overlaps of proton wavefunctions on two 
diabatic electronic potential energy surfaces and the electronic coupling matrix element between them.

By parameterising a simplified model of the diabatic electronic states and their modulation by a donor-acceptor mode, Soudackov and Hammes-Schiffer show that observed H/D kinetic isotope effects for both wild type and mutant SLO can be reproduced throughout the experimentally accessed temperature range. ${ }^{[5}$ This suggests that their approach provides a useful way to interpret these particular experiments. However, it cannot provide a general solution to the PCET problem, because it relies on the reaction conforming to one or other limiting regime (sequential or concerted, adiabatic or non-adiabatic). There are at least two examples in this Discussion of how ET reactions can be in the transition region between the adiabatic and non-adiabatic regimes. ${ }^{35 \mid 45}$ Moreover Kretchmer and Miller have recently used RPMD simulations to show that concerted, sequential electron-proton, and sequential proton-electron transfer pathways all contribute simultaneously to the PCET reaction in iron biimidazoline in liquid acetonitrile $\frac{56}{56}$ and the present contribution by Tiwari and Ensing 5 (which we shall return to discuss in more detail below) uses a simulation to show that even the simplest of ET reactions can have some PCET character. So while analytical expressions for the PCET rate are useful in limiting cases, there are certainly some situations in which one needs to do a simulation to reveal the full complexity of the PCET mechanism.

\section{SAMPLING RARE EVENTS}

Activated chemical reactions involve rare events - fleeting passages through dynamical bottlenecks - and many of the contributions to the present Discussion are concerned with ways to capture them. The probability of observing a rare event per unit time is exponentially small, being proportional to the Boltzmann weight for configurations at the bottleneck. In a complex system, it is impractical to study such events by straightforward molecular dynamics. Instead, one adapts importance sampling to focus on the bottlenecks, and the trajectories that pass through them.

Systematic studies of rare events in complex systems began in the 1970s, with the introduction of the Bennett-Chandler (BC) method. ${ }^{14 / 58}$ This is a two-step procedure that

exploits the correlation function expression for the rate coefficient mentioned in Sec. II. It first estimates a transition state approximation to the rate constant (essentially a free en- 
ergy calculation, which determines the Boltzmann weight), and then estimates a dynamical correction to the approximation (essentially a short-time reactive flux correlation function calculation, which determines the transmission coefficient). The procedure requires a preassigned reaction coordinate, which should ideally be chosen so as to isolate the pertinent dynamical bottleneck(s). When such a reaction coordinate can be pre-assigned correctly, the procedure converts an exponential computational effort into a linear effort.

The $\mathrm{BC}$ method remains useful to this day, and several of the contributions to the present Discussion employ it. For example the paper by Kretchmer and Miller uses it to calculate KC-RPMD electron transfer rates, $\frac{44}{4}$ and the paper by Menzl and co-workers presents an interesting generalisation of the original BC procedure ("S-shooting") and uses it in an impressive calculation of the rate of bubble cavitation in negative pressure water. ${ }^{[5]}$ (Two other contributions to the present Discussion that also focus nucleation are the study of Sanz and co-workers $\frac{60}{60}$ who use a templating method to study the nucleation of crystals in supercooled liquids, and that of Parrinello and co-workers, ${ }^{61}$ who use a variationally enhanced sampling method to calculate the free energy barrier to the nucleation of a liquid droplet in supersaturated vapour.)

Major steps beyond the BC procedure first appeared in the late 1990s, which saw the introduction of methods focussed on trajectories or chains of states rather than states. In doing so, these methods allow one to study rare events in cases where the reaction coordinate cannot be known without first observing trajectories. The methodologies include transition path sampling $62[63$ (TPS) and its extensions that facilitate the calculation of rate constants [transition interface sampling 64 (TIS) and forward flux sampling finite-temperature string method, $\sqrt{66}$ and transition path theory ${ }^{67}$ (TPT, to be distinguished from transition state theory). These methods have now gained significant acceptance, and they feature heavily in the present Discussion (see below).

The 1998 Faraday Discussion 110 included one of the first TPS papers, $\frac{68}{6}$ and the comments in the General Discussions at that meeting highlighted the importance of this new perspective. ${ }^{69}$ One of these comments provides a figure that illustrates the pitfalls of preassigned reaction coordinates, and it also illustrates the distinction between order parameters and reaction coordinates.70 The former characterise basins of attraction, while the latter characterise the mechanism by which trajectories move from one basin to another. It is sometimes assumed that the former can be a good approximation to the latter, but in 
complex systems this assumption is quite often incorrect.

The ideal reaction coordinate is the committor - the probability that a trajectory initiated from a configuration commits to a specified basin. The transition state ensemble (TSE) is the collection of configurations for which the committor is equal to $1 / 2$. In a committor analysis, one tests the accuracy of a putative reaction coordinate by computing distributions of committors. A good reaction coordinate $f(\mathbf{q})$ will define a transition state dividing surface $f(\mathbf{q})=f^{\ddagger}$ on which the configurations $\mathbf{q}$ have a committor distribution sharply peaked at $1 / 2$. A committor analysis requires the shooting of many short trajectories of the kind that are generated in the S-shooting algorithm of Menzl and co-workers. ${ }^{59}$ TPT and the finitetemperature string method are built upon the concept of a committor being the reaction coordinate.

All of these concepts are inherently classical because trajectories are inherently classical, and importance sampling of configuration space is also inherently classical. The melding of concepts such as TPS, TPT, and committor analysis with quantum theory is therefore a challenge, and not possible to do exactly. If one wishes to simulate complex systems in cases where quantum mechanical (zero point energy and tunneling) effects are important, the best one can do is employ a classical model that mimics these effects, such as RPMD. This model possesses the correct thermodynamics and obeys detailed balance consistent with this thermodynamics, which allows it to be combined with essentially all of the modern trajectory-based rare event sampling techniques. Models such as the classical Wigner model that are inconsistent with detailed balance cannot be used in the same way. They might yield reasonable estimates of short-time correlation functions, but such estimates are useful only in cases where rare-event mechanisms are already known.

There are several good examples of the development and application of advanced sampling techniques in the present Discussion, spanning the whole spectrum of TPS, FFS/TIS and TPT methodologies. The first is the paper by Tiwari and Ensing, ${ }^{57}$ which presents a TPS study of $\mathrm{Ru}^{2+}$ to $\mathrm{Ru}^{3+}$ electron transfer in aqueous solution with the electronic aspects of the problem treated using density functional theory (DFT). In the past, DFT treatments of ET have been done by breaking the full redox reaction up into half reactions, but this approach does not provide insight into the mechanism of the full reaction. Remarkably, the finding from TPS of the full reaction is that the (necessarily adiabatic, because of the use of ground state DFT) electron transfer is followed by a proton transfer in the aqueous solvent 
between the two metal ions $\frac{57}{57}$ This discovery of PCET in this simplest of electron transfer reactions is surprising, because it reveals a complexity that is not imagined in the traditional Marcus electron transfer mechanism. Several order parameters are discussed in the paper, but the correct reaction coordinate is hard to discern without a committor analysis. It would be interesting to perform such an analysis to reveal the extent to which the proton position plays a role in the transition state ensemble.

The paper by Vijaykumar and co-workers ${ }^{71}$ explains how the intrinsic rate constants for association and dissociation in a diffusion-influenced reaction can be extracted from a single FFS or TIS simulation. This is clearly a useful simplification, and the results for two isotropic particles undergoing Brownian dynamics, and also for two anisotropic patchy particles, are convincing. This is elegant work that looks as if it could be very useful for future applications in which the intrinsic rate constants are combined with kinetic modelling of more complicated systems with more particles.

An example that involves TPT is presented in the paper by Banisch and VandenEijnden,,$[22$ which develops an algorithm for directly generating loop-erased transition paths (which are shorter than full transition paths and convey the essential mechanism of the transition from reactants to products) for out-of-equilibrium reactions (specifically, Markov chains that are invariant with respect to the ergodic distribution but which do not satisfy detailed balance). The applications to biased random walks on various directed graphs, for which the committor function is relatively easy to calculate, are compelling. However, the new algorithm does not offer any advantage for off-lattice problems with continuous state spaces, for which the flow lines of the current of reactive trajectories are loop-free (see the discussion between P. Bolhuis and E. Vanden-Eijnden in DOI:10.1039/C6FD90075E). In any case, it is pleasing to see that mathematicians are contributing to the development of sampling methods. Another example from the mathematicians is the paper by Schütte and co-workers, ${ }^{73}$ which develops new algorithms for extracting the parameters of an effective Brownian dynamics of a reduced system involving a subset of reaction coordinates from short trajectories of an underlying Brownian dynamics in the full configuration space. Interestingly, this paper also shows that if the full dynamics is ergodic and reversible then these properties are inherited by the reduced dynamics, and that the time scales of the full dynamics are either preserved or underestimated by the reduced dynamics. ${ }^{73}$ 


\section{CHEMICAL APPLICATIONS}

The final theme that has emerged from the contributions to this Discussion is chemistry: a number of the contributions apply various forms of reaction rate theory to chemical problems. These include an ab initio FSSH study of the photorelaxation of imidazole and adenine in water clusters that we have already mentioned, 34 a boxed molecular dynamics calculation of the free energy barrier of the $\mathrm{F}+\mathrm{CD}_{3} \mathrm{CN} \rightarrow \mathrm{DF}+\mathrm{D}_{2} \mathrm{CN}$ reaction in a $\mathrm{CD}_{3} \mathrm{CN}$ solvent, 74 a classical molecular dynamics study of the thermal and collision-induced dissociation of a gas phase peptide, ${ }^{75}$ a combination of DFT, TST, and kinetic modelling applied to the formose reaction in aqueous solution, $\frac{76}{76}$ and two further papers that we shall now end with, both of which address real-world problems (in electrocatalysis and combustion chemistry, respectively).

The paper by Skúlason and co-workers ${ }^{77}$ presents climbing image nudged elastic band calculations of activation energies for the elementary steps in the $\mathrm{CO}_{2}$ reduction reaction and the competing $\mathrm{H}_{2}$ formation reaction on $\mathrm{Pt}(111)$, in the presence of two explicit layers of liquid water. At a (typical) applied potential of $-0.9 \mathrm{eV}$ relative to the standard hydrogen electrode, the rate-limiting activation energy for $\mathrm{CO}_{2}$ reduction is found to be 0.95 $\mathrm{eV}$, whereas the activation energy for $\mathrm{H}_{2}$ formation is only $0.5 \mathrm{eV}$. The activation energies found previously for the same processes on a $\mathrm{Cu}(111)$ electrode are the other way around. This explains why $\mathrm{Cu}$ is a better electrocatalyst for the reduction of $\mathrm{CO}_{2}$ than $\mathrm{Pt}$, a result that cannot be explained using the standard "thermochemical model" that focusses on the energies of reaction intermediates rather than activation energies. ${ }^{78}$ This is clearly important work, and a major result. However, the calculated activation energies are just static potential energy barriers, which neglect the role of bulk water near the metal surface and its fluctuations. Recent work has established that the hydration of metal surfaces can be dynamically heterogeneous and hydrophobic, ${ }^{79}$ so it is possible that in neglecting the fluctuations some important aspects of the physics of the problem have been omitted.

Finally, the paper by Mebel and co-workers ${ }^{13}$ presents the latest chapter in a lengthy effort to obtain temperature- and pressure-dependent rate constants for the (undesirable) formation of polycyclic aromatic hydrocarbons (PAHs) in combustion devices. As we have already mentioned in the introduction, this paper follows the strategy for chemical kinetics set out by Wigner: ab initio calculations are first used to find the energies and partition 
functions of reaction intermediates and transition states, and then elementary reaction rates are combined with a kinetic master equation to solve for the overall $T$ - and $p$-dependent rate constants for various mechanisms of PAH formation and growth. Here the strategy is applied to the formation of indene and its conversion to naphthalene. These are two of the simplest PAHs, but the competing pathways that lead even this far are already rather daunting (see Figs. 7 and 16 in their paper ${ }^{13}$ ), and there is every reason to suppose that they will become even more so for larger PAHs. Real chemical reactions can be awfully complicated!

\section{SUMMARY}

We have seen everything in this Discussion from exploratory applications of new methods to one-dimensional Eckart barriers to state-of-the-art applications of well-established theories to real chemical problems. ${ }^{80}$ The field has clearly progressed a great deal since the 1937 Faraday Discussion, 1 especially in terms of the complexity of the problems that can now be addressed with advanced sampling methods and modern computers. However, there are still some outstanding theoretical challenges, such as the development of a more generally applicable and reliable approach for electronically non-adiabatic dynamics. It would be interesting to look back in another 80 years and see how much progress has been made with that problem.

1 Trans. Faraday Soc., 1938, 34.

2 H. Eyring, ref. 1, pp. 41-48.

3 Undergraduate chemists in Oxford still use Eyring's TST to estimate the rates, kinetic isotope effects, and temperature-dependences of the pre-exponential factors of gas phase reactions, and to infer the likely signs of the volume and entropy of activation for reactions involving ions in aqueous solution, using nothing more than a calculator and a pencil and paper.

4 D. G. Truhlar and B. C. Garrett, Acc. Chem. Res. 1980, 13, 440-448.

5 E. Wigner, J. Chem. Phys. 1937, 5, 720-725.

6 J. C. Keck, J. Chem. Phys. 1960, 32, 1035-1050. 
7 S. Álvarez-Barcia, M-S. Russ, J. Meisner and J. Kästner, Faraday Discuss. 2016, Advance Article.

8 S. Vlasov, P. F. Bessarab, V. M. Uzdin and H. Jónsson, Faraday Discuss. 2016, Advance Article.

9 G. Di Gesù, T. Lelièvre, D. Le Peutrec, and B. Nectoux, Faraday Discuss. 2016, Advance Article.

10 R. Ianconescu and E. Pollak, Faraday Discuss. 2016, Advance Article.

11 W. H. Miller, Faraday Discuss. 1998, 110, 1-21.

12 E. Wigner, ref. 1, pp. 29-41.

13 A. M. Mebel, Y. Georgievskii, A. W. Jasper and S. J. Klippenstein, Faraday Discuss. 2016, Advance Article.

14 D. Chandler, J. Chem. Phys. 1978, 68, 2959-2970.

15 T. Yamamoto, J. Chem. Phys. 1960, 33, 281-289.

16 W. H. Miller, S. D. Schwartz and J. W. Tromp, J. Chem. Phys. 1983, 79, 4889-4898.

17 W. H. Miller, J. Chem. Phys. 1975, 62, 1899-1906.

18 E. Wigner, Z. Phys. Chem. Abt. B 1932, 19, 203-216.

19 W. H. Miller, J. Chem. Phys. 1974, 61, 1823-1834.

20 M. J. Gillan, J. Phys. C 1987, 20, 3621-3641.

21 G. A. Voth, D. Chandler and W. H. Miller, J. Chem. Phys. 1989, 91, 7749-7760.

22 D. Chandler and P. G. Wolynes, J. Chem. Phys. 1981, 74, 4078-4095.

23 T. J. H. Hele and S. C. Althorpe, J. Chem. Phys. 2013, 138, 084108.

24 S. C. Althorpe and T. J. H. Hele, J. Chem. Phys. 2013, 139, 084115.

25 T. J. H. Hele and S. C. Althorpe, J. Chem. Phys. 2013, 139, 084116.

26 T. J. H. Hele and S. C. Althorpe, J. Chem. Phys. 2016, 144, 174107.

27 I. R. Craig and D. E. Manolopoulos, J. Chem. Phys. 2005, 122, 084106.

28 I. R. Craig and D. E. Manolopoulos, J. Chem. Phys. 2005, 123, 034102.

29 R. Collepardo-Guevara, I. R. Craig and D. E. Manolopoulos, J. Chem. Phys. 2008, 128, 144502.

30 R. Collepardo-Guevara, Y. V. Suleimanov and D. E. Manolopoulos, J. Chem. Phys. 2009, 130, 174713.

31 J. O. Richardson and S. C. Althorpe, J. Chem. Phys. 2009, 131, 214106.

32 J. O. Richardson, Faraday Discuss. 2016, Advance Article.

33 J. C. Tully, J. Chem. Phys. 1990, 93, 1061-1071. 
34 R. Szabla, R. W. Góra, M. Janicki and J. Šponer, Faraday Discuss. 2016, Advance Article.

35 J. Spencer, L. Scalfi, A. Carof and J. Blumberger, Faraday Discuss. 2016, Advance Article.

36 There has been one attempt to include nuclear quantum effects in FSSH by combining it with RPMD, but this combination does not appear to have been widely adopted. See: P. Shushkov, R. Li and J. C. Tully, J. Chem. Phys. 2012, 137, 22A549.

37 E. R. Bittner and P. J. Rossky, J. Chem. Phys. 1995, 103, 8130-8143.

38 C. Zhu, S. Nangia, A. W. Jasper and D. G. Truhlar, J. Chem. Phys. 2004, 121, 7658-7670.

39 G. Granucci and M. Persico, J. Chem. Phys. 2007, 126, 134114.

40 J. E. Subotnik and N. Shenvi, J. Chem. Phys. 2011, 134, 024105.

41 W. H. Miller, Faraday Discuss. 2016, Advance Article.

42 S. J. Cotton and W. H. Miller, J. Chem. Phys. 2016, 145, 144108.

43 N. Bellonzi, A. Jain and J. E. Subotnik, J. Chem. Phys. 2016, 144, 154110.

44 J. S. Kretchmer and T. F. Miller III, Faraday Discuss. 2016, Advance Article.

45 J. R. Duke and N. Ananth, Faraday Discuss. 2016, Advance Article.

46 A. R. Menzeleev, F. Bell and T. F. Miller III, J. Chem. Phys. 2014, 140, 064103.

47 N. Makri, Faraday Discuss. 2016, Advance Article.

48 P. Walters and N. Makri, J. Phys. Chem. Lett. 2015, 6, 4959-4965.

49 T. J. H. Hele and N. Ananth, Faraday Discuss. 2016, Advance Article.

50 H.-D. Meyer and W. H. Miller, J. Chem. Phys. 1979, 70, 3214-3223.

51 H.-D. Meyer and W. H. Miller, J. Chem. Phys. 1979, 71, 2156-2169.

52 G. Stock and M. Thoss, Phys. Rev. Lett. 1997, 78, 578-581.

53 G. Stock and M. Thoss, Adv. Chem. Phys. 2005, 131, 243-376.

54 S. Habershon and D. E. Manolopoulos, J. Chem. Phys. 2009, 131, 244518.

55 A. V. Soudackov and S. Hammes-Schiffer, Faraday Discuss. 2016, Advance Article.

56 J. S. Kretchmer and T. F. Miller III, Inorg. Chem. 2016, 55, 1022-1031.

57 A. Tiwari and B. Ensing, Faraday Discuss. 2016, Advance Article.

58 C. H. Bennett, in Algorithms for Chemical Computations, ACS Symposium Series No. 46, edited by R. E. Christofferson (American Chemical Society, Washington, DC, 1977), pp. 63-97.

59 G. Menzl, A. Singraber and C. Dellago, Faraday Discuss. 2016, Advance Article.

60 J. R. Espinosa, P. Sampedro, C. Valeriani, C. Vega and E. Sanz, Faraday Discuss. 2016, Advance Article. 
61 P. M. Piaggi, O. Valsson and M. Parrinello, Faraday Discuss. 2016, Advance Article.

62 C. Dellago, P. G. Bolhuis, F. S. Csajka and D. Chandler, J. Chem. Phys. 1998, 108, 1964-1977.

63 P. G. Bolhuis, D. Chandler, C. Dellago and P. L. Geissler, Ann. Rev. Phys. Chem. 2002, 53, 291-318.

64 T. S. van Erp, D. Moroni and P. G. Bolhuis, J. Chem. Phys. 2003, 118, 7762-7774.

65 R. J. Allen, D. Frenkel and P. R. ten Wolde, J. Chem. Phys. 2006, 124, 024102.

66 E. Weinan, W. Ren and E. Vanden-Eijnden, J. Phys. Chem. B 2005, 109, 6688-6693.

67 E. Vanden-Eijnden, Lect. Notes Phys. 2006, 703, 439-478.

68 P. G. Bolhuis, C. Dellago and D. Chandler, Faraday Discuss. 1998, 110, 421-436.

69 See the comments by Bolhuis, Chandler and Dellago in the General Discussion Faraday Discuss. 1998, 110, 477-520.

70 See the comment by Chandler on p. 370 of the General Discussion Faraday Discuss. 1998, 110, $347-378$.

71 A. Vijaykumar, P. G. Bolhuis and P. R. ten Wolde, Faraday Discuss. 2016, Advance Article.

72 R. Banisch and E. Vanden-Eijnden, Faraday Discuss. 2016, Advance Article.

73 W. Zhang, C. Hartmann and C. Schütte, Faraday Discuss. 2016, Advance Article.

74 M. O'Connor, E. Paci, S. McIntosh-Smith and D. R. Glowacki, Faraday Discuss. 2016, Advance Article.

75 R. Spezia, A. Martin-Somer, V. Macaluso, Z. Homayoon, S. Pratihar and W. L. Hase, Faraday Discuss. 2016, Advance Article.

76 J. Proppe, T. Husch, G. N. Simm and M. Reiher, Faraday Discuss. 2016, Advance Article.

77 J. Hussain, H. Jónsson and E. Skúlason, Faraday Discuss. 2016, Advance Article.

78 J. K. Norskov, J. Rossmeisl, A. Logadóttir, L. Lindqvist, J. R. Kitchin, T. Bligaard and H. Jónsson, J. Phys. Chem. B 2004, 108, 17886-17892.

79 D. T. Limmer, A. P. Willard, P. Madden and D. Chandler, Proc. Nat. Acad. Sci. USA 2013, 110, 4200-4205.

80 There are two further contributions to the present Discussion which do not fit into any of the themes we have covered. The first describes a new method for performing quantum scattering calculations [J. Tennyson, L. K. McKemmish and T. Rivlin, Faraday Discuss. 2016, Advance Article], and the second compares optical and chemical driving of molecular machines [R. D. Astumian, Faraday Discuss. 2016, Advance Article]. 